\section{A superfamily of ion channels}

SIR-Recent studies have revealed structural similarities among voltage-gated cation channels. It would be interesting to know whether some of the secondmessenger-gated channels are similar in structure to voltage-gated channels. We report here that a second-messenger-gated channel, the bovine rod photoreceptor cyclic GMP-gated channel ${ }^{1}$, belongs to the same superfamily as voltage-gated cation channels.

Like voltage-gated potassium channel polypeptides $^{2-5}$, the cyclic GMP-gated channel polypeptide resembles one of the

four internally homologous domains of the alpha-subunit of voltage-gated sodium or calcium channels ${ }^{6-9}$. The six hydrophobic sequences $(\mathrm{H} 1-\mathrm{H} 6)$ and an $\mathrm{S} 4$ sequence that is between $\mathrm{H} 3$ and $\mathrm{H} 4$ of the cyclic GMP-gated channel can be aligned with the corresponding sequences of potassium channels and each of the four domains of sodium and calcium channels (see figure). The probability of this occurring by chance is negligible, suggesting that cyclic GMP-gated and voltage-gated cation channels are related in structure.

The presence of an $\mathrm{S} 4$ sequence in the
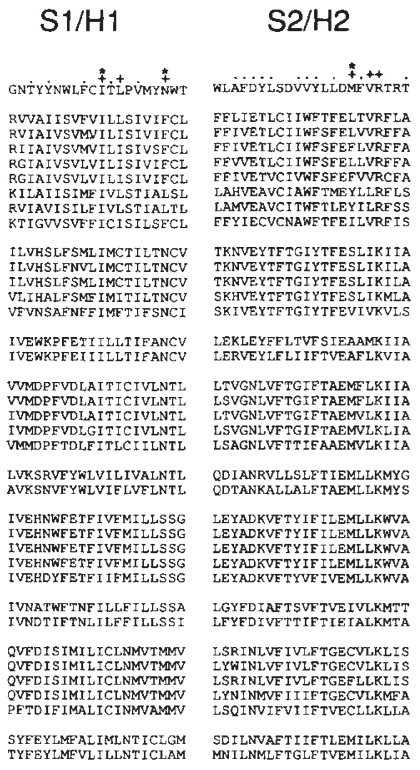

$$
\mathrm{S} 3 / \mathrm{H} 3
$$
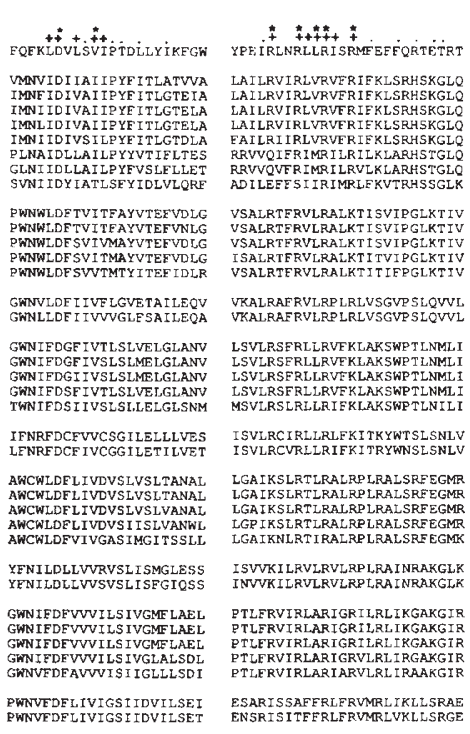

$\mathrm{S} 5 / \mathrm{H} 4$

S4

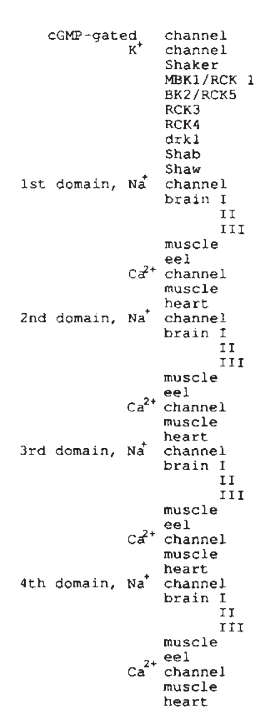
ISNLVMYYIITIHWNACVYFST $\mathrm{H} 5$
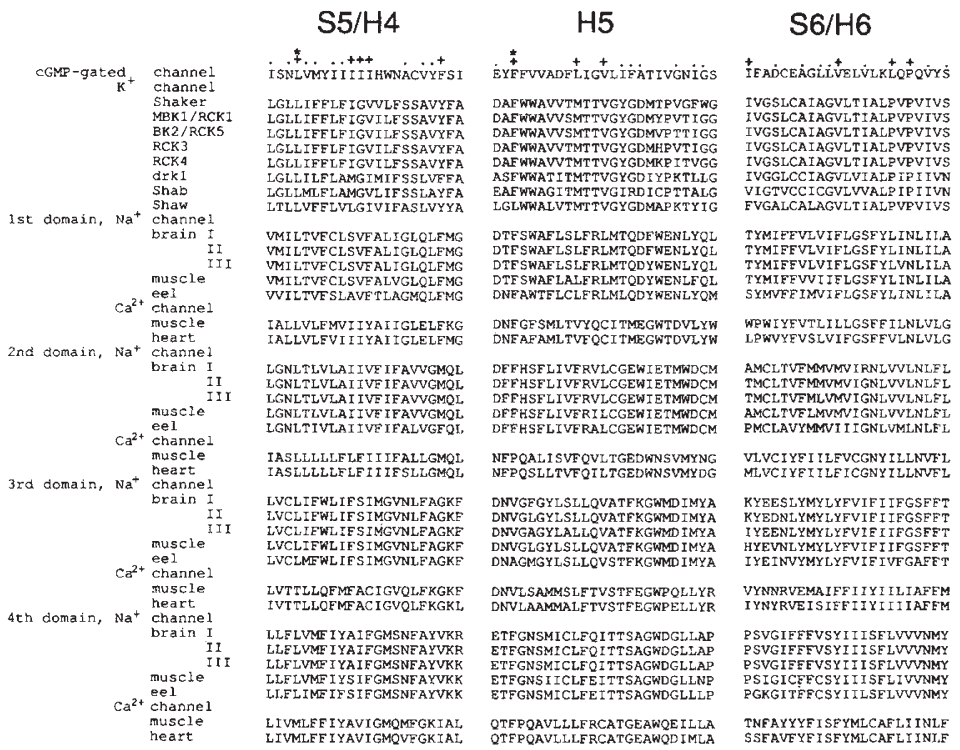
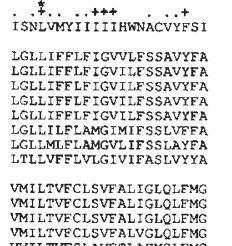

IALLVLEMVIIYAIIGLELFKG
IALLVLFVI IIYAI IGLELFMG

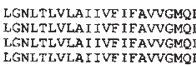

LGNLTZVLAIIVF IFAVVGMQ

IASLLLLLFLFIIIFALLGMQL
IASLLLLLFLFIITFSLLGQL

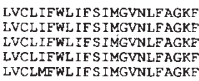

LVTTLLQFMFACIGVQLEKGKF
IVTTLLQFMFAC IGVQLFKGKL

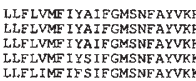

LIVMLFFIYAVIGMOMEGKIAI
LIVMLF IYAVIGMQVEGKIAL
Sequence alignment of the cyclic GMP-gated channel and voltage-gated potassium, sodium and calcium channels. Crosses, residues in the cyclic GMP-gated channels found in at least 8 of the 36 sequences of voltage-gated cation channels. Dots, residues found in less than 8 , but at least one of these 36 sequences. Asterisks, residues in the cyclic GMP-gated channel that are identical to the corresponding residues in four or more of the nine groups of voltage-gated channel sequences (the group of Shaker and RCK1,3,4,5, and each of the four domains of the sodium or calcium channel). The probability of finding the same level of sequence identity as observed for all 12 residues marked by asterisks is $2.3 \times 10^{-56}$. Details of the sequence alignment and statistical treatment are available from the authors on request. cyclic GMP-gated channel polypeptide is interesting, as the S4 sequences in voltage-gated channels have been proposed to serve the function of voltage sensors. Unlike voltage-gated channels, the cyclic GMP-gated channel requires the binding of three or more molecules of cyclic GMP to become activated, though the gating shows weak voltage dependence (see ref. 1 for futher references). The conservation of the S4 sequence in this secondmessenger-gated channel could reflect a requirement for the close packing of the protein interior.

Ponder and Richards ${ }^{10}$ have proposed that the basic architecture of a globular protein is largely determined by the 'tertiary template', the core structure formed by buried residues that are inaccessible to solvent. Only a few particular combinations of amino acids fulfill the criteria for the tertiary template for a given globular protein $^{10,11}$. By analogy, the tertiary template of an ion channel could correspond to those residues that do not interact with water or lipids, or ions in the pore; these residues form the core structure and are important in establishing the basic architecture of the channel. Before the structure of a channel is determined, its tertiary template can not be identified. But on the basis of their conservation, it seems likely that some of the hydrophobic residues, and perhaps even some of the basic residues that are highly invariant among the cyclic GMP-gated and voltage-gated cation channels, correspond to part of this tertiary template. Thus, the $\mathrm{S} 4$ sequence probably arose in an ancestral channel. In addition to its postulated function as a voltage sensor for some of the descendant voltage-gated channels, it probably forms part of the core structure of both voltagegated and cyclic GMP-gated channels. LILY YEH JAN YUH NUNG JAN

Howard Hughes Medical Institute and Departments of Physiology and Biochemistry, University of California, San Francisco, San Francisco 94143, USA
1. Kaupp, U.B. et al. Nature 342, 762-766 (1989).
2. Schwartz, T.L. et al. Nature 331, 137-142 (1988)
3. Stühmer, W. et al. EMBO J. 8, 3235-3244 (1989).
4. Frech, G.C. et al. Nature 340, 642-645 (1989).
5. Butler, A. et al. Science 243, 943-947 (1989).
6. Noda, M. et al. Nature 312, 121-127 (1984).
7. Trimmer, J.S. et al. Neuron 3, 33-49(1989).
7. Trimmer, J.S. et al. Neuron 3, 33-49(1989).
8. Tanabe, T. et al. Nature 328, 313-318 (1987).
9. Mikanni, A. et al. Nature 340, 230-233 (1989).
10. Ponder, J.W. \& Richards, F.M. J. molec. Biol. 193, 775- $791(1987)$.
11. Bowie, J.U. et al. Science 247, 1306-1310 (1990).

\section{Scientific Correspondence}

Scientific Correspondence is intended to provide a forum in which readers may raise points of a scientific character. They need not arise out of anything published in Nature. In any case, priority will be given to letters of fewer than 500 words and five references. 